\title{
The parallel dynamics of drift wave turbulence in the WEGA stellarator
}

\author{
S Marsen, M Endler, M Otte and F Wagner \\ Max-Planck-Institut für Plasmaphysik, EURATOM Association, \\ Wendelsteinstraße 1, 17491 Greifswald, Germany \\ E-mail: stefan.marsen@ipp.mpg.de
}

\begin{abstract}
The three-dimensional structure of turbulence in the edge (inside the last closed flux surface) of the WEGA stellarator is studied focussing on the parallel dynamics. WEGA as a small stellarator with moderate plasma parameters offers the opportunity to study turbulence with Langmuir probes providing high spatial and temporal resolution. Multiple probes with radial, poloidal and toroidal resolution are used to measure density fluctuations. Correlation analysis is used to reconstruct a 3D picture of turbulent structures. We find that these structures originate predominantly on the low field side and have a three-dimensional character with a finite averaged parallel wavenumber. The ratio between parallel and perpendicular wavenumber component is in the order of $10^{-2}$. The parallel dynamics are compared at magnetic inductions of $57 \mathrm{mT}$ and $500 \mathrm{mT}$. At $500 \mathrm{mT}$, the parallel wavelength is in the order of the field line connection length $2 \pi R / t$. A frequency resolved measure of $k_{\|} / k_{\theta}$ shows a constant ratio in this case. At $57 \mathrm{mT}$ the observed $k_{\|}$is much smaller than at $500 \mathrm{mT}$. However, the observed small average value is due to an averaging over positive and negative components pointing parallel and antiparallel to the magnetic field vector.
\end{abstract}

\section{Introduction}

The understanding of turbulence, the major cause of losses in magnetic confinement devices, is a key issue on the way to a fusion reactor. In recent years it has been shown that small machines too, with dimensions and plasma parameters far from fusion relevant values, can contribute significantly to this research area [1]. One of the advantages of small devices with moderate plasma parameters is the applicability of Langmuir probes for fluctuation measurements providing an excellent spatial and temporal resolution. The plasma parameters in these experiments are moderate enough to place probes stationary in the plasma, even in the region of closed flux surfaces. This work focuses on presenting details on the three-dimensional structure of turbulence and its dynamics parallel to the magnetic field vector in the edge region of a stellarator plasma. In a stellarator very detailed information on the parallel dynamics can be obtained since the magnetic field configuration is usually known very precisely and can be mapped experimentally as will be shown later.

By edge region, we mean the region just inside the last closed flux surface (LCFS) close to the scrape-off layer (SOL). Most previous experimental works presenting information on the parallel dynamics of turbulence focussed on the SOL. However, theoretical models comparing turbulence in regions of open and closed field lines 
predict turbulence in the edge to differ from the SOL in its driving mechanism [2]. In the edge region periodic boundary conditions, introduced by the magnetic field topology, lead to the physical consequence, that the wavenumber component parallel to the magnetic field $k_{\|}$cannot vanish for disturbances of finite size. In this case, the electrostatic potential $\tilde{\phi}$ is coupled to the electron pressure $\tilde{p}_{e}$ through parallel forces mediated by parallel currents $\tilde{J}_{\|}$, where $\left(^{\sim}\right)$ denotes the fluctuating part of the quantity. This type of dynamics is referred to as drift wave turbulence. In contrast, in the SOL, the field lines end on divertor plates or limiters. The periodic boundary conditions from the edge therefore do not apply and turbulence with $k_{\|}=0$ in the plasma is allowed. This type of dynamics is called interchange turbulence.

Absolute values of $k_{\|}$observed in the SOL of tokamaks and stellarators vary from values vanishing within the error bars $[3,4,5]$ up to finite values in the order of $k_{\|} \approx(0.5 \ldots 10) t / R[6,7,8]$, where $t$ is the rotational transform and $R$ is the major radius of the device. A common result of experiments is that fluctuations appear as localized events of finite lifetime, which are strongly elongated along the magnetic field [9]. A strong anisotropy exists with parallel correlation lengths being at least two orders of magnitude larger than in the poloidal direction. A detailed study of the parallel dynamics of turbulence in the edge region has been performed at the TJ-K torsatron [10], where turbulence is clearly dominated by drift wave dynamics, as it is also the case for the WEGA stellarator [11].

The results presented in this paper further support the picture of drift wave dynamics dominating turbulence in the edge of toroidal devices, and we shall present details on its parallel dynamics.

The ideal experimental setup to study the spatio-temporal structure of turbulence would be a 3D grid of measurement points as indicated in figure 1. Due to the strong elongation of structures along the magnetic field vector $\mathbf{B}$, the grid distance in toroidal direction may be much larger $(\mathrm{O}[m])$ than in the radial and poloidal direction $(\mathrm{O}[\mathrm{cm}])$. Such a setup, if sampled at an appropriate temporal resolution, would be able to reflect the real spatio-temporal structure of fluctuation events. However, due to limited experimental access, such an approach to the 3D structure of turbulence has to our knowledge not been realized in a fusion device. Typical experimental setups are limited to two sampling points in the toroidal direction $[4,5,8]$. A more sophisticated approach has been realized in the TJ-K torsatron [10] and in a similar manner in WEGA. The approach used in this work is a $2 \mathrm{D}$ matrix of sampling points in the radial-poloidal plane ( $r-\theta$ plane), supported by an additional single reference point which is separated from the grid in the toroidal direction (black dots in figure 1). Information about the parallel dynamics of turbulence is obtained by placing the reference point on a field line intersecting the grid after a defined connection length ( $L_{c}$, approximately the toroidal probe separation). Applying this two-point approach together with a heuristic model as the basis for the interpretation of the results [5] one can already conclude about the 3D structure of turbulence. The model will be introduced in section 2. In section 3 the experimental setup and the results from experimental field line mapping will be presented. Results on the parallel dynamics of turbulence in WEGA in different operational regimes will be presented in section 4 and finally discussed in section 5 . 


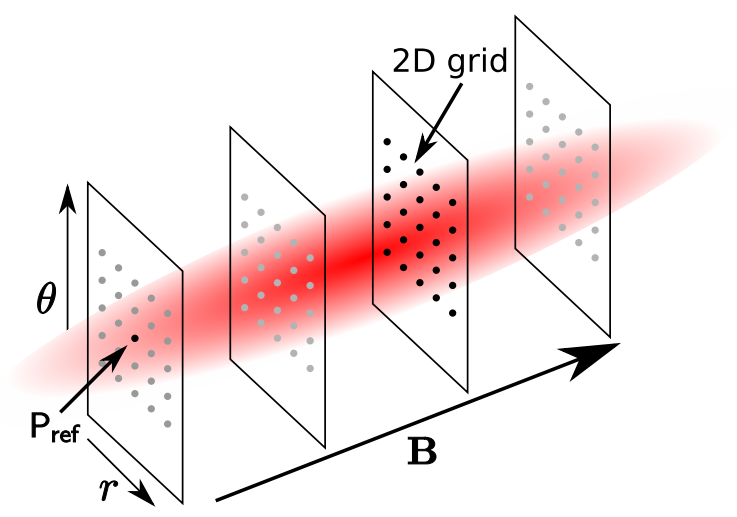

Figure 1. Experimental approach to study the spatio-temporal structure of turbulence. A $3 D$ grid of points sampling elongated filaments would be the ideal diagnostic.

\section{Heuristic model of three-dimensional drift wave turbulence}

For simplification, the approach given in figure 1 is reduced to 2D geometry in the poloidal-toroidal plane $(\theta-\varphi$ plane) and a coherent wave is considered first. The geometry is depicted in figure 2 (a). The position $P_{0}$ marks the point where a field line, starting at the reference probe $\left(P_{\text {ref }}\right)$, intersects the grid. Data are analysed by calculating the cross-correlation function $(\mathrm{CCF})$ between time traces taken with the reference probe and the corresponding time traces from the individual points of the grid. The position where the highest correlation is found within the matrix is of particular interest. This may, in general, be shifted with respect to the intersection point $P_{0}$ by a certain length $\delta_{\theta}(t)$. The highest cross-correlation for zero time lag $\tau$ occurs at $P_{1}$ (corresponding to $\delta_{\theta, 0}$ ), which is aligned on the wavefront crossing $P_{r e f}$. In the case of a finite $k_{\|}, P_{1}$ is displaced by a distance of $\delta_{\theta, 0}$ with respect to $P_{0}$. The method to derive information about $k_{\|}$from this two point measurement in the toroidal direction has been introduced by Ritz et al [6]. The ratio $k_{\|} / k_{\theta}$ is given by the inclination angle $\vartheta$, and can thus be derived from the knowledge of $\delta_{\theta, 0}$ and the parallel probe separation length $L_{c}$ :

$$
k_{\|} / k_{\theta}=\delta_{\theta, 0} / L_{c}=\tan \vartheta .
$$

$k_{\|}$can also be determined directly from the phase shift $\alpha$ between $P_{\text {ref }}$ and $P_{0}$ :

$$
k_{\|}=\alpha_{r e f, 0} / L_{c} .
$$

The parallel phase velocity $v_{\|}$of the wave can be derived from the time a wavefront needs to propagate along $\mathbf{B}$ from $P_{\text {ref }}$ to $P_{0}$. In the CCF between these two points this is the time lag of maximum cross-correlation $\tau_{1}$ :

$$
v_{\|}=L_{c} / \tau_{1} .
$$

The relations up to now apply to a planar wave of a given frequency and wavenumber. However, the considerations may be transferred to turbulent density fluctuations which typically appear as localized events with a finite correlation length in all three dimensions. Such turbulent structures are in general advected by the background poloidal $\mathbf{E} \times \mathbf{B}$ velocity. The following description will consider only the 


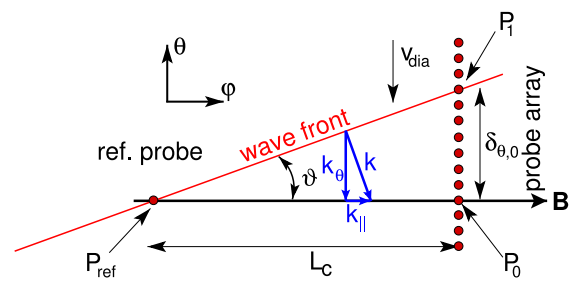

(a) Wave picture

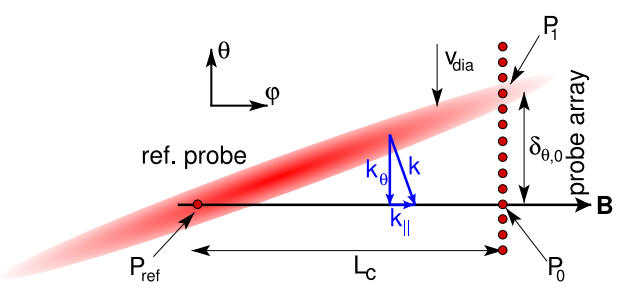

(b) Blob picture

Figure 2. a) A coherent drift wave, with a wavefront slightly tilted with respect to $\mathbf{B}$ is sampled by probes at two toroidal positions separated by the connection length $L_{c}$. $P_{\text {ref }}$ and $P_{0}$ are aligned on a connecting field line. $P_{\text {ref }}$ and $P_{1}$ are aligned on a wavefront. b) In case of turbulence no clear wavefront exists, but structures elongated along $\mathbf{B}$. In this case $P_{1}$ is the point of maximum correlation at $\tau=0$.

self-consistent dynamics of turbulent structures. This is valid, as long as the radial electric field is either negligible or leads to an unsheared $\mathbf{E} \times \mathbf{B}$ velocity profile.

The toroidal correlation length is expected to be about two orders of magnitude longer than in the poloidal direction $\left(k_{\|} / k_{\theta} \approx 10^{-2}\right)$. Hence, the structures, colloquially dubbed as 'blobs', are strongly elongated in the parallel direction and represent fluctuation events as illustrated in figure $2(\mathrm{~b})$. In this representation $v_{\|}$is an averaged phase velocity. (2) becomes the parallel dispersion relation $k_{\|}(\nu)$ with the cross-phase spectrum $\alpha_{\text {ref,0 }}(\nu)$ between $P_{\text {ref }}$ and $P_{0}$. Following Ritz et al [6] an averaged cross-phase between two signals 1 and 2 can be defined:

$$
\bar{\alpha}_{1,2}=\int\left|\Psi_{1,2}(\nu)\right| \alpha_{1,2}(\nu) d \nu / \int\left|\Psi_{1,2}(\nu)\right| d \nu
$$

where $\left|\Psi_{1,2}(\nu)\right|$ is the absolute cross-power spectrum. An average parallel wavenumber $\bar{k}_{\|}$corresponds to $\bar{\alpha}$ following (2).

Assuming the wavefront of all contributing Fourier components to point in the same direction $\left(k_{\|} / k_{\theta} \neq f(\nu)\right)$, the complete structure is tilted by an angle $\vartheta$ with respect to the magnetic field. Evidence for the validity of this representation can be obtained from the cross-phase spectra along the legs of the triangle defined by $P_{\text {ref }}, P_{0}$ and $P_{1}$. $P_{\text {ref }}$ and $P_{1}$ are assumed to be aligned on the same wavefront for all scales $\mathbf{k}(\nu)$. Therefore, the cross-phase $\alpha_{\text {ref, } 0}(\nu)$ should tend to zero, in contrast to a finite phase shift for $P_{0}$ which is displaced normal to the so-called wavefront. The normal distance from the wavefront is equal for the paths $\overrightarrow{P_{\text {ref }}, P_{0}}$ and $\overrightarrow{P_{1}, P_{0}}$. Hence, similar cross-phases are expected along the legs of the triangle in figure 2.

In contrast to plane waves, where a velocity component parallel to the wavefronts cannot be defined, a 'blob' can have velocity components in all directions. Therefore, an observed $L_{c} / \tau_{1}$ can be the sum of the projection of the poloidal velocity $v_{\|}^{\prime}=$ $\left(L_{c} / \delta_{\theta, 0}\right) v_{\theta}$ and a true parallel component of the 'blob' velocity $v_{\|}^{\prime \prime}$ :

$$
v_{\|}=L_{c} / \tau_{1}=v_{\|}^{\prime}+v_{\|}^{\prime \prime} .
$$

If we now proceed to multiple such blob structures with finite lifetimes, different cases have to be distinguished considering the statistical spatial distribution of structures. A preferred toroidal phase velocity is inherent in this representation because all $k_{\|}$components of the spectrum are assumed to point in the same direction. An additional component of the parallel dynamics is the growth and thereby the 
spatial expansion of structures with time. The correlation maximum within the grid undergoes a temporal evolution giving information about this statistical distribution. For fluctuations arising randomly at any point in the toroidal direction, the correlation function would, on average, see a structure which appears between the two toroidal positions under observation. The highest correlation in the matrix would, in this case occur at zero time lag at $P_{1}$.

A different concept is that fluctuations appear preferably in a certain region along the magnetic field line. This assumption is not unrealistic because a strong poloidal asymmetry of fluctuation properties is a common observation in fusion devices $[12,13,14,15]$. Poloidal asymmetries in the edge are always linked to asymmetries along the magnetic field line as the rotational transform connects different poloidal regions along a magnetic field line. However, a preferred occurrence of fluctuations at a certain point, and an expansion from this point would lead to a finite time lag of maximum cross-correlation between reference and grid if the propagation times from the 'starting point' to reference and grid are not equal. This time lag (called $\tau_{2}$ subsequently) may occur when the correlation maximum is at any position in the matrix along the path of the structure.

A more complicated picture results if the assumption of the ratio $k_{\|} / k_{\theta}$ being constant for all frequency components does not hold. An example is an equally distributed spectrum of parallel wavenumbers without a preferred direction. This may result in vanishing values of $\delta_{\theta, 0}$ and $\tau_{1}$. In this case no detailed information about the parallel dynamics of turbulence can be retrieved from the correlation function.

We now turn from general geometrical considerations to specific properties of drift waves. Due to the finite $k_{\|}$of the drift wave, $\delta_{\theta, 0}$ is expected to show finite values in WEGA. A simple estimation of the smallest values of $\delta_{\theta, 0}$ to be expected shall be given here. $k_{\|}$can be estimated based on a fundamental assumption which underlies the Wakatani-Hasegawa drift wave turbulence model [16]. $k_{\|}$is assumed to be in the order of $t / R$ where $R$ is the major plasma radius. The corresponding wavelength $2 \pi R / t$ is called the field line connection length, which for a rational surface with a toroidal modenumber of $m=1$ is the length of a field line before it closes upon itself. The poloidal wavenumber is estimated by $k_{\theta} \approx 0.15 / \rho_{s} . \rho_{s}=\sqrt{m_{i} k_{B} T_{e}} / e B$ is the so-called drift scale. Simulations of drift wave turbulence in stellarator geometry show the shoulder of the spectral power of $k_{\theta}$ to be in the range between $0.1 / \rho_{s}$ and $0.2 / \rho_{s}[17]$ as it is typically observed in experiments [18]. For WEGA parameters this estimation leads to $k_{\|} \approx t / R \approx 0.3 \mathrm{~m}^{-1}$ and an expected perpendicular displacement of $\delta_{\theta, 0}=k_{\|} \rho_{s} / 0.15 \cdot L_{c} \approx 0.5 \mathrm{~cm}$ with $\rho_{s} \approx 0.1 \mathrm{~cm}$.

\section{Experimental setup}

WEGA is a classical five period $l=2$ stellarator. It is a small experiment (major radius $R=72 \mathrm{~cm}$, aspect ratio $\approx 7$ ) with moderate plasma parameters. Typical densities and electron temperatures are in the range of $n_{e} \lesssim 5 \cdot 10^{18} \mathrm{~m}^{-3}$ and $T_{e}$ up to some $10 \mathrm{eV}$, respectively [19]. WEGA provides a very flexible magnetic field configuration with a toroidal field of up to $B_{0}=0.9 \mathrm{~T}$. The rotational transform is determined by the ratio between the toroidal and helical field coil current $\left(t \propto\left(I_{H} / I_{T}\right)^{2}\right)$ and can be varied in a range from zero to about 1 . All results presented in this paper have been obtained using helium as working gas in a field configuration with $t_{0}=0.224$ on the magnetic axis. The plasma is heated by ECRH at $2.45 \mathrm{GHz}$ (maximum $26 \mathrm{~kW}(\mathrm{cw})$ ) [20] or $28 \mathrm{GHz}$ (maximum $10 \mathrm{~kW}(\mathrm{cw})$ ) leading to more or less fixed working points 


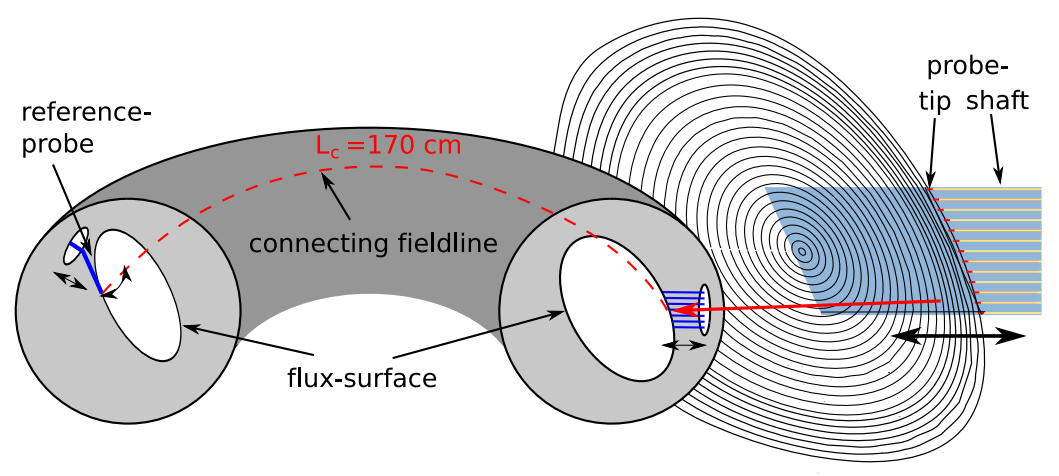

Figure 3. Probe arrangement for toroidally resolved measurements. A poloidal array of 13 probes samples the shaded region on the $r-\theta$-plane in a radial scan. A single stationary reference probe can be placed on a field line intersecting the sampling region of the array displaced by a connection length of $L_{c}=170 \mathrm{~cm}$.

for the magnetic induction at $B_{0}=57 \mathrm{mT}$ and $B_{0}=500 \mathrm{mT}$, respectively. These two operational regimes of WEGA will be labelled low field and high field operation subsequently. In the high field operation densities for the plasma conditions under consideration were typically by about one order of magnitude higher than at low field, while the temperatures were comparable.

\subsection{Langmuir probe arrangement}

The moderate plasma parameters allow the use of Langmuir probes along the whole plasma cross-section. The common practice of operating the probes only at single working points for fluctuation measurements was applied here, namely all results in this paper show measurements of the ion saturation current, which is assumed to be proportional to density fluctuations neglecting temperature fluctuations $\left(\tilde{I}_{s a t, i} \propto \tilde{n}_{e}\right)$. It is well known that this approach may lead to errors in estimating absolute values of turbulent transport which is affected by temperature fluctuations [21]. But considering the statistical properties of fluctuations, like correlation functions and Fourier spectra, results from $\tilde{I}_{s a t, i}$ measurements have been shown to be in good agreement with independent methods including temperature fluctuations [22].

The technical realization of the experimental approach to $3 \mathrm{D}$ turbulence given in section 1 is shown schematically in figure 3 . Two probe systems are installed at WEGA. A single probe placed on a 2D movable manipulator acts as the reference probe for toroidally resolved measurements. The actual spatial information is obtained from a poloidal array of probes with probe tips aligned to the flux surfaces. The array of 13 probes with a poloidal distance of $5 \mathrm{~mm}$ is mounted on a fast reciprocating manipulator performing a radial scan in about $1 \mathrm{~s}$ covering the poloidal cross-section marked in figure 3. An essential point in order to get information about the parallel dynamics of turbulence is that the reference probe can be placed in such a manner, that a field line starting at its tip intersects the cross-section covered by the array in a well-defined connection length (here $L_{c}=170 \mathrm{~cm}$ ).

Using the reference probe together with the array it is possible to reconstruct averaged turbulent structures in the sampling region. To this end the time traces taken during a radial scan of the array are divided into subwindows to get a radial 
resolution. A scan is typically divided into 20-30 slices of 20-40 ms each (3000060000 samples), resulting in a radial resolution of $\delta r \approx 2-4 \mathrm{~mm}$. Thus a grid of sampling points is produced, where individual time traces are taken. This grid will be referred to as the matrix in the following. Turbulent structures are now reconstructed by calculating the cross-correlation function $(\mathrm{CCF})$ between each subwindow of the matrix and simultaneously sampled subwindows of data from the reference probe. To make data from different regions along the pressure gradient comparable, all subwindows are normalized to their fluctuation amplitude prior to the correlation analysis.

A point to be briefly discussed here is the unavoidable perturbation of the plasma by the probes. Although probe arrays are widely used for spatially resolved studies of plasma turbulence it is recently discussed, in how far turbulence is affected by the probes penetrating the plasma. In our case, this has been checked looking at the evolution of fluctuations properties measured by the stationary reference probe while the array is entering the plasma. It could be shown that the relative fluctuation amplitude as well as the spectral properties remained unaffected.

\subsection{Field Line Tracing}

A prerequisite for the construction of a three-dimensional picture of turbulence is a precise knowledge of the magnetic topology. The statistical analysis of raw data is based on a two point measurement with probes aligned on a connecting field line. As detailed above, the expected effect is a small shift of turbulent structures with respect to the connecting field line. Field line tracing calculations were used to determine constellations where connecting field lines exist. However, relying on these calculations might introduce an error, which is hard to determine, since the absolute coil currents as well as the absolute position of both probe systems with respect to the coil system need to be known precisely. The effect of small misalignments of probes on the results of toroidally resolved measurements has been discussed by Thomsen et al [23] for the case of a time dependent field perturbation in a tokamak.

However, in a stellarator the magnetic field configuration can be determined experimentally, and at low $\beta$ ( $\beta$ was below $10^{-4}$ for the experimental conditions considered here) the vacuum magnetic configuration is not noticeably altered during plasma operation. Using experimental field line mapping, only the reproducibility of probe positions (about $\pm 1 \mathrm{~mm}$ in our case) and the magnetic configuration $(t)$ determined by the coil currents have to be considered. The magnetic configuration expressed in terms of the relative error of the rotational transform is reproducible by $\Delta t / t<0.25 \%$. The error in determining the intersection point of the connecting field line resulting from this uncertainty is below $1 \mathrm{~mm}$ in the poloidal plane.

Experimental field line mapping is based on the electron beam technique which is also used for flux surface measurements [24, 25]. To this end the reference probe was replaced by an electron gun injecting an electron beam into the vacuum magnetic field parallel or antiparallel to B. The beam energy was set to $30 \mathrm{eV}$ by the accelerating voltage in the gun. This relatively low beam energy was chosen in order to minimize the $\nabla \mathbf{B}$ and curvature drift. The perpendicular drift along the toroidal probe separation length of $1.7 \mathrm{~m}$ is below $1 \mathrm{~mm}$ for electrons at $30 \mathrm{eV}$, even at an induction of only $50 \mathrm{mT}$. The gun was placed roughly at a position where numerical calculations with the W7-code [26] predicted the existence of a connecting field line to the matrix. The beam with a width of $\approx 2 \mathrm{~mm}$ followed a field line and could 


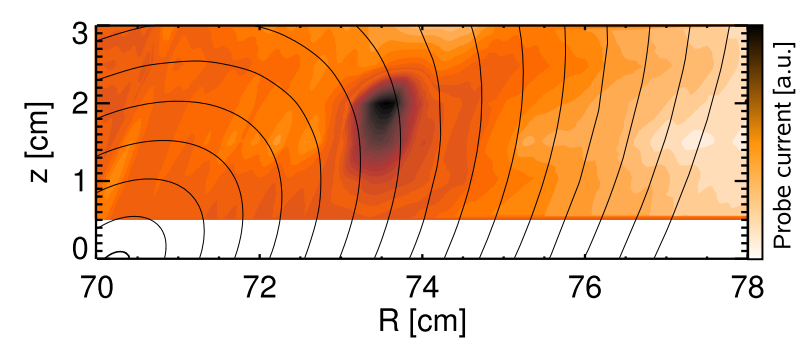

Figure 4. Visualization of the electron beam detected by the probe array. The intensity represents the amplitude of the electron current collected by the grounded probe tips Only the part of the matrix is shown, where the beam was observed. The dark region marks the highest intensity where the beam intersects the poloidal plane covered by the array. The picture shows an example, where the intersection point is deep in the plasma core.

be visualized in a background gas at a low neutral pressure (argon at a pressure of $p_{N} \approx 10^{-3} \mathrm{~Pa}$ ) to see its intersection with the matrix.

In order to determine the exact intersection point, the beam was finally detected by the probe array itself. To this end, the probe tips were grounded over a shunt resistor. The electron current collected by the probes was measured in a radial scan of the array. Figure 4 shows the amplitude of the detected current for a configuration where the connecting field line was located close to the plasma centre. The intersection point of the electron beam can be clearly seen as a strong local increase in the signal intensity. With the help of this field line mapping technique different connecting field lines were determined. Two positions of the reference probe lying approximately on the same flux surface were selected for the plasma experiments. The first one intersects the matrix in the equatorial plane at $\mathrm{z}=0$, the second one near the lower end of the matrix at $\mathrm{z}=-2.5 \mathrm{~cm}$. The considered flux surface is about $5 \mathrm{~mm}$ inside the LCFS. A comparison between experimental mapping and calculations shows a discrepancy of $\gtrsim 5 \mathrm{~mm}$ for the intersection point. The reason for this discrepancy is probably a sum of several small systematic errors. The absolute positioning of the reference probe and the array as well as erroneous absolute coil currents contribute to this error. Simulations have shown, that a relative error of only $\approx 2 \%$ in one magnetic field component (i.e. toroidal or helical field) can explain the observed discrepancy. This shows the importance of the experimental field line mapping because the expected effect of $\delta_{\theta, 0} \approx 1 \mathrm{~cm}$, is in the order of this discrepancy. The results presented below are based on the experimental field line mapping shown in this section.

\section{Experimental results}

\subsection{Summary of general characterisation of turbulence in WEGA}

The poloidal probe array has been used to study the general character of turbulence in WEGA and its poloidal dynamics. Results from the first experimental campaign have been published previously [11] leading to the conclusion, that turbulence in WEGA is dominated by drift wave dynamics. Some of the main results characterizing turbulence in WEGA and leading to this conclusion are summarized here.

- Turbulent broadband power spectra are observed, which are flat up to some 10 
$\mathrm{kHz}$ and decay following a power law for higher frequencies.

- Typical correlation times representing the averaged lifetime of turbulent structures are in the order of $\tau_{L} \approx 100 \mu \mathrm{s}$.

- Strongest density and potential fluctuations are observed in the region of the steepest density gradient. That is turbulence in WEGA is driven by the pressure gradient providing the free energy as it is the case for drift wave turbulence.

- The cross-phase between density and potential perturbations is below $\pi / 4$ on all scales of significant fluctuation power. Assuming adiabatic electrons, density and potential fluctuations associated with the drift wave are in phase. However, any dissipative effect in the parallel electron dynamics introduces a finite phase shift. In WEGA, mainly collisional effects cause the observed phase shift.

- The poloidal correlation length $d_{\theta}$, representing the averaged structure size, was found to scale nearly linearly with the inverse magnetic induction. It was about $d_{\theta} \approx 2 \mathrm{~cm}$ for high field $(500 \mathrm{mT})$ and $d_{\theta} \approx 14 \mathrm{~cm}$ for low field $(57 \mathrm{mT})$. These variations fit well with theoretical prediction of drift wave turbulence models predicting $\rho_{s} \propto 1 / B$ to be a characteristic scaling length for the shoulder of $k_{\theta}$ spectra [27].

- The poloidal velocity inside the LCFS points in the electron diamagnetic drift direction. $v_{\theta}$ inside the LCFS was also nearly proportional to $1 / B$, i.e. several $100 \mathrm{~m} / \mathrm{s}$ at high field and several $\mathrm{km} / \mathrm{s}$ at low field. The poloidal $\mathbf{E} \times \mathbf{B}$ velocity was negligible here. This is again an indicator of drift wave turbulence as the poloidal phase velocity of drift waves is the electron diamagnetic drift velocity $v_{\text {dia,e }} \propto 1 / B$. A change of sign of $v_{\theta}$ in the SOL caused by $E \times B$ drift was observed in the high field case.

An important point for the following results on the parallel dynamics is that the poloidal $\mathbf{E} \times \mathbf{B}$ drift in the edge of of WEGA is negligible compared with the electron diamagnetic drift velocity. Therefore, the self-consistent dynamics of turbulent structures can be observed in WEGA without $\mathbf{E} \times \mathbf{B}$ advection.

\subsection{Toroidally resolved experiments at high field}

4.2.1. Temporal evolution of turbulent structures. The matrix decomposition of a radial scan was used to reconstruct the radial-poloidal correlation function in the poloidal plane covered by the array as explained in section 3. Figure 5 shows the relative correlation between the reference probe and individual points in the matrix. Each plot is a snapshot of the correlation function for a fixed time lag. The dot at $R=78.9 \mathrm{~cm}, z=-2.5 \mathrm{~cm}$ marks the experimentally determined intersection point of the field line starting at $P_{\text {ref }}$, where the diameter of the dot corresponds roughly to the measurement error determined by the spatial resolution provided by the probe array. Information about the dynamics of turbulence in the poloidal plane can be gained from the series of snapshots for increasing time lags in figure 5 . At $\tau<-80 \mu \mathrm{s}$ a structure enters the observation window at the upper boundary $(z=+2.5 \mathrm{~cm})$. With increasing time lag the correlation amplitude at the centre of the structure continuously increases until a certain point and finally decreases again, thus reflecting the lifetime of the structure. During its lifetime the structure propagates in the poloidal direction following the flux surface shape (solid black lines in figure 5). A movie showing the complete temporal evolution of the CCF is available online as a multimedia attachment. The structure is elongated in the poloidal direction by a 


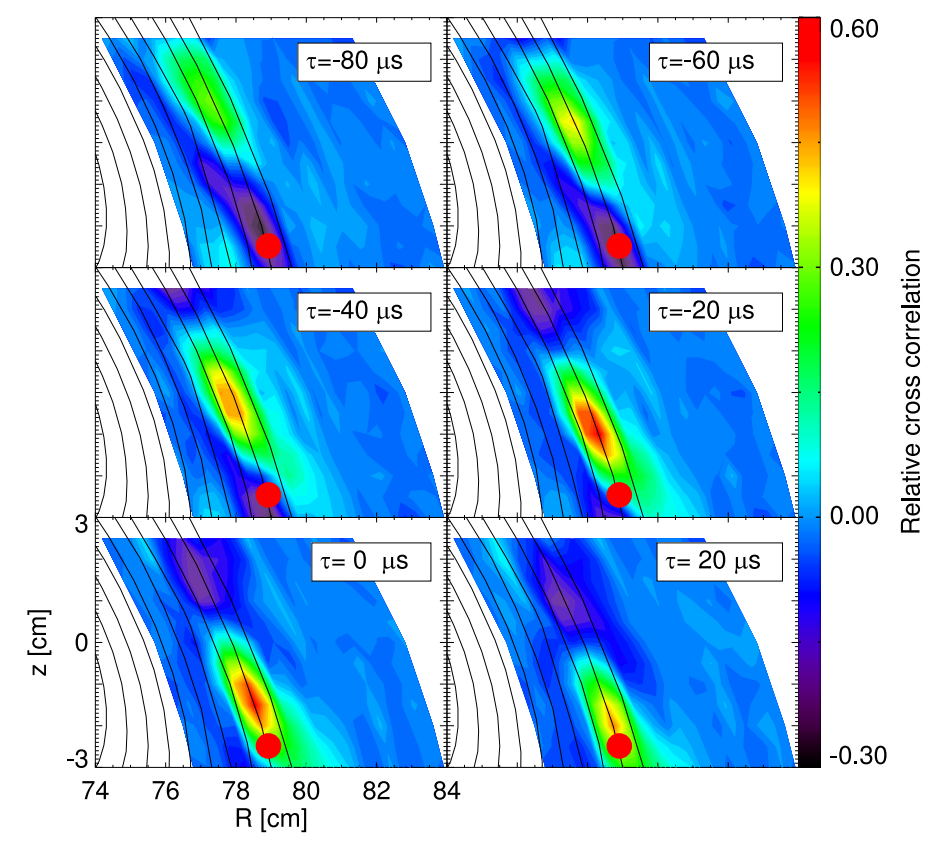

Figure 5. Cross-correlation function between the data from the reference probe and individual subwindows in the matrix for a He discharge at $B \approx 500 \mathrm{mT}$, $t \lesssim 0.25, n_{e} \approx 10^{18} \mathrm{~m}^{-3}$ and $T_{e} \lesssim 10 \mathrm{eV}$. The dot at $R=78.9 \mathrm{~cm}, z=2.5 \mathrm{~cm}$ marks the intersection point of the connecting field line. The temporal evolution of the observed structures is illustrated as a series of snapshots in the $R-\theta-$ plane for different time lags $\tau$. The structure propagates along a flux surface (black lines) in electron diamagnetic drift direction. The figure does not represent the dynamics of an individual fluctuation event but a statistical average. A movie showing the complete temporal evolution of the CCF is available online as a multimedia attachment.

factor of about 2 , hence the poloidal correlation length $(2 \mathrm{~cm})$ is longer than the radial correlation length $(\approx 1 \mathrm{~cm})$.

The colour coding in figure 5 yields the time lag of maximum correlation between $\tau=-20 \ldots 0 \mu \mathrm{s}$. A different representation of the temporal evolution of structures is shown in figure 6 . It shows the highest correlation amplitude of all points of the matrix as a function of the time lag. Additionally, the poloidal distance of this maximum from the intersection point of the connecting field line is plotted. The temporal evolution of the correlation maximum clearly shows the growth of structures up to a relative correlation of about $60 \%$ and the following decay. The inclination of $\delta_{\theta}(\tau)$ reflects the poloidal velocity of structures. $v_{\theta}$ was constant in the observed window. A linear fit resulted in $v_{\theta} \approx 300 \mathrm{~m} / \mathrm{s}$, in good agreement with the electron diamagnetic drift velocity derived from stationary plasma parameters.

The most interesting information that can be gained from figure 6 comes from the time lag of maximum correlation, labelled $\tau_{2}$, which clearly deviated from zero. The maximum was found at $\tau_{2}=(-12 \pm 2.2) \mu \mathrm{s}$, where the given error is a measure for the statistical scatter of data among individual discharges. By definition, a negative sign of $\tau_{2}$ means, that structures were on average first observed at the matrix and delayed by $\tau_{2}$ at $P_{\text {ref }}$. This result gives evidence that fluctuation events did not arise 


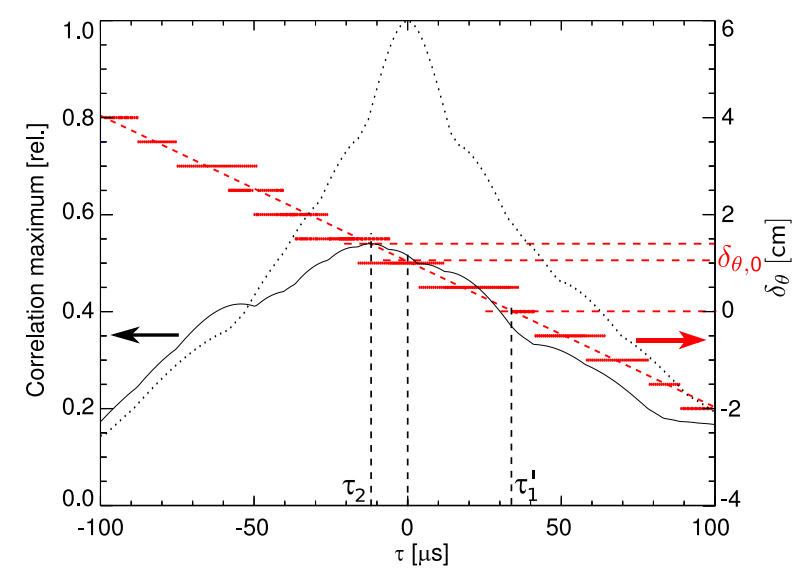

Figure 6. Temporal evolution of the correlation maximum in figure 5. (black -) Correlation maximum in the centre of the structure, (red - - - ) displacement of the maximum from the intersection point. The highest correlation is observed at $\tau_{2}$. At $\tau=0$ the centre of the structure is displaced from the intersection point by $\delta_{\theta, 0} \approx 1 \mathrm{~cm}$. (black, .....) Envelope of the poloidaltemporal correlation function, measured only by the array on the flux surface under consideration.

randomly at any position. They preferably appeared closer to the toroidal position of the matrix and expanded from there. No information about the symmetry of expansion could be gained from these experiments because no further measurement points in the toroidal direction but the reference probe were available. Information about the average parallel wavenumber can be gained from both figure 5 and 6 . To this end we consider the centre of the structure at zero time lag. It was found to be displaced from the intersection point by $\delta_{\theta, 0}=(1.1 \pm 0.3) \mathrm{cm}$. Hence, in the blob representation presented above the structure was tilted with respect to the connecting field line by a small angle $\vartheta$ (see section 2). Following (1) the ratio between the average parallel and perpendicular wavenumber was: $\bar{k}_{\|} / \bar{k}_{\theta}=\tan \vartheta=(0.6 \pm 0.17) \cdot 10^{-2}$. A quantitative evaluation of absolute values of $k_{\|}$from cross-spectral analysis will be given in the subsequent section.

The next step was to look at the correlation between two points aligned on the same field line $\left(P_{\text {ref }}\right.$ and $\left.P_{0}\right)$. Figure 7 shows their cross-correlation function together with the average of their auto-correlation functions. Both functions show nearly the same width and shape. The cross-correlation maximum was relatively small $(\approx 40 \%)$ because the structure was already collapsing when it passed $P_{0}$ at a time lag of $\tau_{1}=(24.3 \pm 2.6) \mu \mathrm{s}$. Following $(3), \tau_{1}$ determines the averaged parallel phase velocity of structures: $v_{\|}=L_{c} / \tau_{1}=(7 \pm 0.74) \cdot 10^{4} \mathrm{~m} / \mathrm{s}$. Hence, the parallel phase velocity of structures had a preferred direction, pointing from $P_{\text {ref }}$ towards $P_{0}$. As pointed out in section $2, v_{\|}$is the sum of two contributions. The contribution of $v_{\theta}$ was: $v_{\|}^{\prime}=v_{\theta}\left(L_{c} / \delta_{\theta, 0}\right)=(4.6 \pm 1.3) \cdot 10^{4} \mathrm{~m} / \mathrm{s}$. The intrinsic parallel blob velocity component $v_{\|}^{\prime \prime}$ was estimated to be the ion sound velocity. $c_{s}$ is, for the large scales under consideration $\left(k_{\theta} \rho_{s}<<1\right)$, the upper limit at which a density perturbation associated with a drift wave can propagate along a field line. $c_{s}$ as derived from stationary plasma parameters was $\approx 1.5 \cdot 10^{4} \mathrm{~m} / \mathrm{s}$. Within the error bars $v_{\|}$was the sum of $v_{\|}^{\prime}$ and $v_{\|}^{\prime \prime}=c_{s}$. Due to the parallel dynamics of structures $\tau_{1}$ is not 


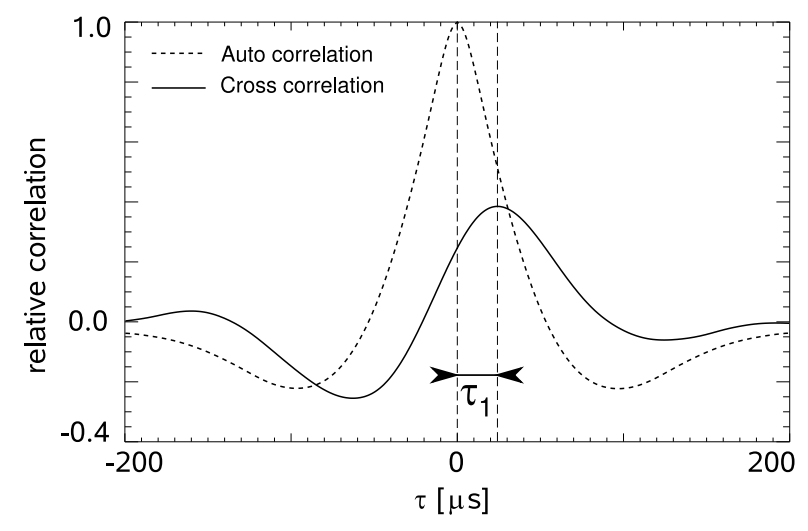

Figure 7. Temporal cross-correlation function between data from $P_{\text {ref }}$ and $P_{0}$ (solid line). The dashed line reflects the averaged auto-correlation function for both points. The time lag of maximum cross-correlation, $\tau_{1}$, corresponds to a finite parallel phase velocity of structures.

necessarily the time when their centre passes $P_{0}$. In figure 6 it can be seen that the structure passes the intersection point at a time lag of $\tau_{1}^{\prime} \approx 34 \mu \mathrm{s}$. This results in $v_{\|}^{\prime}=L_{c} / \tau_{1}^{\prime}=5 \cdot 10^{4} \mathrm{~m} / \mathrm{s}$, in good agreement with the estimation from $v_{\theta}\left(L_{c} / \delta_{\theta, 0}\right)$.

4.2.2. Parallel and perpendicular dispersion relation. More quantitative results on $k_{\|}$and about the validity of the model description in section 2 could be derived from cross-spectral analysis. Poloidally resolved measurements in WEGA have shown that the spectral power of turbulence in $k_{\theta}-\nu$-space is distributed in a narrow band around the drift wave dispersion relation. This allows the interpretation of crossphase spectra from poloidally separated points as the dispersion relation following $k_{\theta}(\nu)=\alpha(\nu) / \Delta y$, where $\Delta y$ is the spatial separation of measurement points. This relation can be generalized for any spatial direction. In the triangle $\left\{P_{\text {ref }}, P_{0}, P_{1}\right\}$ in figure 2, the cross-phase between $P_{\text {ref }}$ and $P_{0}$ then reflects the parallel dispersion relation, $k_{\|}(\nu)=\alpha_{\|}(\nu) / L_{c} . \quad k_{\theta}$ is derived from $P_{1}$ and $P_{0}$. If the assumptions made in the description of the 'blob' representation are valid, $k_{\theta}(\nu)$ and $k_{\|}(\nu)$ should coincide. Furthermore, the phase shift between $P_{\text {ref }}$ and $P_{1}$ should vanish because both points are assumed to be aligned on the same wavefront for all Fourier components contributing to the turbulent spectrum. Figure 8 (a) shows the cross-phase spectra, together with the corresponding coherence spectra for all three legs of the triangle. For the given probe distance, a significant coherence for this type of discharge was observed for frequencies up to $\nu \approx 8 \mathrm{kHz}$. In this range the good agreement between poloidal and parallel cross-phase is apparent. Both show a similar shape. The vanishing crossphase between $P_{\text {ref }}$ and $P_{1}$ verifies the assumption that in the given frequency range of significant coherence both probe tips were aligned along a wavefront. Since the ratio $k_{\|} / k_{\theta}$ was independent of the frequency, the Fourier components of the turbulent broadband spectrum of wavenumbers $\mathbf{k}$ pointed in the same direction. A small phase shift along the line, claimed to be a wavefront, was remanent. This was on the one hand due to the typical jitter in the turbulent spectra. On the other hand the limited spatial resolution did not allow a perfect alignment of two probes to a wavefront. $P_{1}$ was solely the point of highest correlation in the matrix at $\tau=0$. 


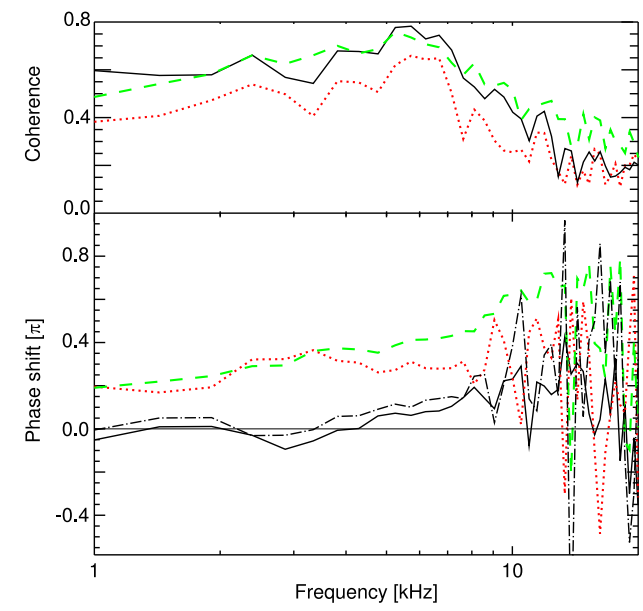

(a) high field

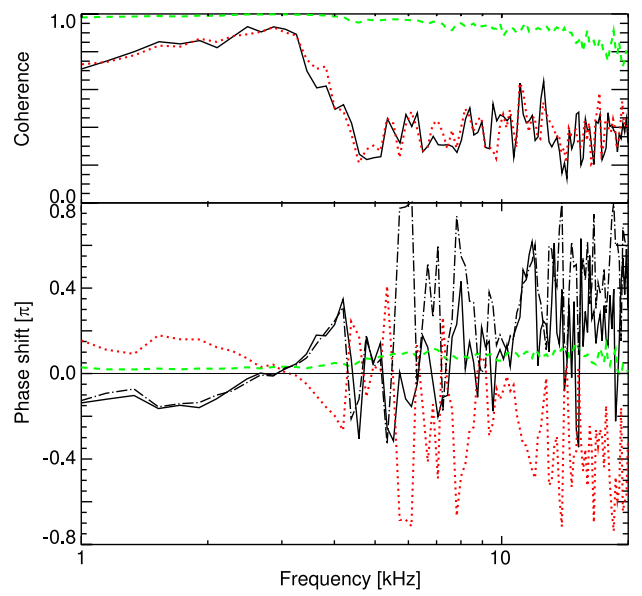

(b) low field

Figure 8. Coherence (top) and cross-phase (bottom) spectra calculated along the legs of the triangle in the $\varphi-\theta$-plane defined by $P_{\text {ref }}, P_{0}$ and $P_{1}$. ( - , black) $\alpha\left(P_{\text {ref }} \rightarrow P_{1}\right)$, so-called wavefront. $(\cdots$, , red $) \alpha\left(P_{\text {ref }} \rightarrow P_{0}\right)$, parallel wavenumber component. (---, green) $\alpha\left(P_{1} \rightarrow P_{0}\right)$, poloidal wavenumber component. (_- - - black) parallel - poloidal component. Data was taken from a high field (a) and a low field (b) discharge.

To come to a quantification of $k_{\|}$the cross-power weighted average parallel wavenumber was calculated using (2) and (4). The parallel phase spectrum from figure 8 (a) yielded an average cross-phase of $\bar{\alpha}=0.869 \pm 0.067$, corresponding to an average parallel wavenumber of $\bar{k}_{\|}=\bar{\alpha} / L_{c}=(0.51 \pm 0.04) \mathrm{m}^{-1}$. This result is in the order of $t / R \approx 0.3 \mathrm{~m}^{-1}$, implying that the averaged parallel wavelength was in the order of the system size defined by the field line connection length.

\subsection{Toroidally resolved experiments at low field}

The parallel turbulence dynamics was also studied for the low field case. The ratio $I_{H} / I_{T}$ and hence the rotational transform could be kept constant in order to achieve the same connecting field lines as in the high field case.

4.3.1. Temporal evolution of turbulent structures. The general information about the temporal evolution of structures that can be gained from the CCF between the reference probe and the matrix was, at first sight, comparable to the results from high field discharges. Structures, propagating in the electron diamagnetic drift direction during the process of growth and decay were observed. Figure 9 shows a snapshot of the $\mathrm{CCF}$ at $\tau=0$. Due to the large poloidal correlation length, which was already observed in poloidally resolved measurements, the structure is not fully contained in the observation window. The structure was more strongly elongated in the poloidal direction relative to the radial size than at the high field experiments. The evaluation of $\tau_{2}$ from the maximum of the CCF in the matrix showed as a universal feature of WEGA that fluctuation events preferably arise closer to the matrix. $\tau_{2}$ was of the same order as in the high field case and also negative. Identical experiments were undertaken at an inverted magnetic field to give evidence for the universal character 


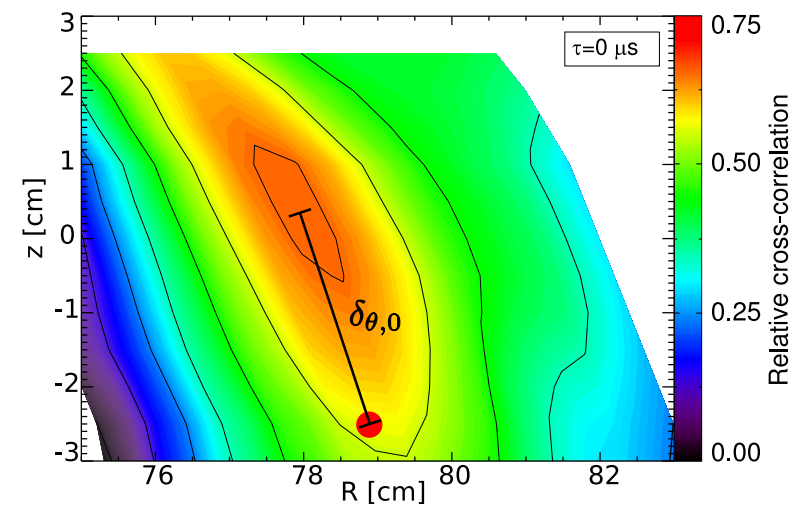

Figure 9. Snapshot of the cross-correlation function between the reference probe and the matrix at $\tau=0$ for a discharge at low magnetic field $(57 \mathrm{mT})$

of this observation. The magnetic topology remains constant if both, $I_{T}$ and $I_{H}$ are inverted. The result of these experiments was that $\tau_{2}$ remained negative. Hence, structures arose on average closer to the matrix and expanded towards the reference probe, independently of the orientation of the magnetic field vector.

The centre of the structure in figure 9 is shifted versus the intersection point by $\delta_{\theta, 0}=(2.2 \pm 0.7) \mathrm{cm}$, yielding a ratio of $\bar{k}_{\|} / \bar{k}_{\theta}=(1.3 \pm 0.4) \cdot 10^{-2}$.

A parallel phase velocity of $v_{\|}=(2.1 \pm 0.46) \cdot 10^{5} \mathrm{~m} / \mathrm{s}$ could be deduced from these experiments for the low field case. However, the two contributions of $v_{\|}$could not be discriminated within the error bars for the low field case, since $v_{\|} \gg c_{s}$. But $v_{\|}$was still in the order of the estimation from $v_{\theta}\left(\bar{k}_{\theta} / \bar{k}_{\|}\right)$.

4.3.2. Parallel and Perpendicular Dispersion Relations. The relation of crossphase spectra in the triangle $\left\{P_{\text {ref }}, P_{0}, P_{1}\right\}$ should clarify, whether the same model description is valid for both low and high fields. Figure 8 (b) shows the result of the cross-spectral analysis. The phase shift in the poloidal direction is rather small, as expected since the poloidal separation of the probes under consideration $\left(\delta_{\theta, 0} \approx 2 \mathrm{~cm}\right)$ was much smaller than the poloidal correlation length $\left(d_{\theta} \approx 14 \mathrm{~cm}\right)$. This is also reflected by the high coherence, which was close to unity in the observed frequency range. A completely different shape of both coherence and cross-phase spectrum was observed in the parallel direction. In striking contrast to the high field case the coherence between toroidally separated probes was almost completely lost for frequencies higher than $\nu \approx 4 \mathrm{kHz}$, although both probes showed similar auto-power spectra, which were flat up to $\nu \gtrsim 10 \mathrm{kHz}$. In the frequency range of high coherence the parallel cross-phase showed a well-defined shape, but changed sign from negative values at very low frequencies to positive values at higher frequencies. By definition, a negative phase shift represents a wavenumber component pointing from the matrix to the reference probe and vice versa. The conclusion to be drawn from this result is that the observed turbulent structures at the low field in WEGA cannot be described in the same manner as in the high field case. The 'blob' representation, describing turbulence as an interaction of Fourier components with constant ratio $k_{\|} / k_{\theta}$ (parallel wavefronts), does not hold anymore.

However, the average parallel cross-phase was formally derived for the spectra 
shown in figure 8 (b). It was rather small and corresponded quite well to the average poloidal cross-phase. The spectra yielded average parallel and poloidal wavenumbers of $\bar{k}_{\|}=(0.085 \pm 0.035) \mathrm{m}^{-1}$ and $\bar{k}_{\theta}=(0.08 \pm 0.009) \mathrm{cm}^{-1}$, respectively. Hence, the ratio $\bar{k}_{\|} / \bar{k}_{\theta}$ agreed satisfactorily with the results from the correlation analysis $\left(\bar{k}_{\|} / \bar{k}_{\theta}=\delta_{\theta, 0} / L_{c}\right)$.

\section{Discussion}

The results presented give a detailed insight into the parallel dynamics of drift wave turbulence under largely different discharge conditions in the WEGA stellarator. A heuristic model based on drift wave theory was used to interpret the results which relied on only two measurement points in the toroidal direction. The results agreed well with the assumptions made in the model and thus the expectations for drift wave turbulence. At $500 \mathrm{mT}$, the averaged parallel wavenumber, which was expected to be finite as we are dealing with drift waves, was found to be $\bar{k}_{\|}=(0.51 \pm 0.04) \mathrm{m}^{-1}$. As expected for the large scales under consideration $\left(\bar{k}_{\theta} \rho_{s} \ll 1\right)$ the parallel phase velocity was far below the Alfvén velocity, which is given as the upper limit of $v_{\|}$for small scales where a coupling between drift and Alfvén dynamics becomes important, as e.g. observed in TJ-K [10]. For the given discharge conditions the Alfvén velocity was in the order of $v_{A} \approx 10^{6} \mathrm{~m} / \mathrm{s} \gg v_{\|}$for both low and high fields.

Poloidally resolved measurements with the probe array showed structures appearing randomly at any point in the sampling region. This spatial homogeneity was obviously valid only in a limited section like the sampling region of the array and not for a complete poloidal turn. In toroidally resolved measurements a clear inhomogeneity was observed which was independent of the magnetic field direction (finite $\tau_{2}$ with constant sign). The observation of a finite negative value of $\tau_{2}$, however, does not allow to give quantitative information about the expansion time of structures. It only shows that there was an asymmetry and fluctuations arose on average closer to the array than to the reference probe. A possible interpretation of this result is that turbulent structures preferably arise on the low field side of the torus, i.e. in the region of unfavourable magnetic curvature. Field lines departing from the matrix pass the reference probe on their way to regions of favourable curvature regions. This is of course in contrast to the argument of drift waves to be the dominating instability driving turbulence in WEGA. Drift waves are not driven by magnetic curvature but by the density gradient. However, fully developed turbulence in toroidal devices is based on, in general, both curvature and density gradient driven instability mechanisms [28]. Although drift wave dynamics is obviously dominating in WEGA, curvature effects may also play a role for turbulence activity.

A strong qualitative difference in the turbulence dynamics between low and high field operation was observed. At high field, a vanishing cross-phase between the two points with maximum correlation at $\tau=0$ was observed. This allows the interpretation, that all Fourier components contributing to the turbulent broadband spectrum have parallel wavevectors, i.e. the ratio $k_{\|} / k_{\theta}$ was constant in the frequency range of high coherence.

At low field, the spectrum of $k_{\|}$showed positive and negative components in the frequency range of high coherence standing for wavenumber components pointing parallel and antiparallel to the magnetic field. The small averaged value of $\bar{k}_{\|}=$ $(0.085 \pm 0.035) \mathrm{m}^{-1}$ results from an averaging over components of different signs with 
a kind of skewness in the spectrum towards positive values. In order to explain the actual shape of the $k_{\|}$spectrum, it is helpful to go back to the picture of planar waves. From a simple geometrical treatment it can be shown, that due to periodicity constraints in a toroidal device only parallel wavenumbers of $k_{\|}=1 / R(t n+m)$ are allowed to exist, where $m$ and $n$ are the integer toroidal and poloidal modenumbers, respectively. In general, both $m$ and $n$ may be positive or negative. But they are associated with the wavenumber component in the corresponding spatial direction and thus the respective phase velocity component. The poloidal phase velocity of drift waves points in the electron diamagnetic drift direction, as was observed in our experiments. This leads to a constraint in the sign of $n$ which is defined to be positive in our case. Small values of $k_{\|}<t / R\left(\approx 0.3 \mathrm{~m}^{-1}\right.$ in our case) are therefore only allowed for negative $m$ corresponding to $k_{\|}$components pointing antiparallel to the magnetic field. The appearance of negative $k_{\|}$components may, in this sense, be understood as a necessary part of the parallel turbulence dynamics at low field in WEGA in order to explain the small value of $\bar{k}_{\|}$.

The reason for the striking difference between low and high field operation of WEGA cannot be clarified here, but is possibly related to the observed perpendicular scaling lengths, since at low field the poloidal correlation length is in the order of the plasma dimensions.

Future experiments shall clarify, whether the two types of parallel dynamics observed at low and high magnetic fields obey a continuous transition by operating at intermediate magnetic inductions.

\section{References}

[1] Stroth U et al . Phys. Plasmas, 11:2558-64, 2004.

[2] Ribeiro T T and Scott B. Plasma Phys. Control. Fusion , 47:1657-79, 2005.

[3] Rudyj A et al. In 16th EPS Conf. on Plasma Phys. and Contr. Fusion (Venice, Italy), volume 13B, pages 27-30, 1989

[4] Bleuel J et al . New J. Phys., 4:38, 2002.

[5] Thomsen H et al. Phys. Plasmas, 9:1233-40, 2002.

[6] Ritz Ch P et al . Rev. Sci. Instrum. , 59:1739-44, 1988.

[7] Winslow D L et al . Rev. Sci. Instrum. , 68:396-99, 1997.

[8] Bengtson R D et al . Contrib. Plasma Phys., 38(special issue):104-9, 1998.

[9] Endler M. J. Nucl. Mat., 266-269:84-90, 1999.

[10] Mahdizadeh N et al . Plasma Phys. Control. Fusion , 49:1005-17, 2007.

[11] Marsen S et al . Contrib. Plasma Phys., 48:467-72, 2008.

[12] LaBombard B and Lipschultz B. Nucl. Fusion, 27:81-99, 1987.

[13] Fenzi C et al. Nucl. Fusion, 40:1621-26, 2000.

[14] Kirnev G S et al. Nucl. Fusion, 45:459-67, 2005.

[15] Ramisch M et al. In 35. EPS Conf. on Plasma Phys. and Contr. Fusion (Hersonissos, Crete, Greece), volume 32, pages P-4.028, 2008.

[16] Wakatani M and Hasegawa A. Phys. Fluids, 27:611-18, 1984.

[17] Kendl A et al. Phys. Plasmas, 10:3684-91, 2003.

[18] Liewer P C. Nucl. Fusion, 25:543-621, 1985.

[19] Horvath K et al . Plasma Phys. Control. Fusion , 48:315-23, 2006.

[20] Podoba Y Y et al . Phys. Rev. Lett. , 98:255003, 2007.

[21] Schubert M et al . Rev. Sci. Instrum. , 78:053505, 2007.

[22] Pfeiffer U et al. Contributions to Plasma Physics, 38(special issue):134-44, 1998.

[23] Thomsen H et al . Contrib. Plasma Phys., 41:530-36, 2001.

[24] Jaenicke R et al. Nucl. Fusion, 33:687-704, 1993.

[25] Otte M and Lingertat J. In 29th EPS Conf. on Plasma Phys. and Contr. Fusion (Montreux, Switzerland), volume 26B, page 5.036, 2002.

[26] Werner A. private communication, 2006.

[27] Terry P W and Diamond P H. Phys. Fluids, 28:1419-39, 1985. 
Parallel dynamics of turbulence in WEGA

[28] Scott B D. New J. Phys., 4:52, 2002. 